\title{
Responsabilité fondée sur l'absence de diagnostic prénatal
}

\author{
La Cour suprême du canton de Berne a été amenée à juger si l'absence d'avorte- \\ ment et la naissance d'un enfant handicapé consécutives à la décision de ne pas \\ effectuer de diagnostic prénatal donnait droit à l'octroi d'une indemnité à titre de \\ réparation morale (action pour wrongful life) [1]*.
}

\section{Ursina Pally Hofmann}

Responsable suppléante du service juridique de la FMH

\footnotetext{
* Les références se trouveront sous www.bullmed.ch $\rightarrow$ Numéro actuel ou $\rightarrow$ Archives $\rightarrow 2013 \rightarrow 15$.
}

Correspondance: Dr iur. Ursina Pally Hofmann Avocate

Service juridique de la FMH Elfenstrasse 18

CH-3000 Berne15

Tél. 0313591111

Fax 0313591112

ursina.pally[at]fmh.ch

\section{Faits et droit}

La femme enceinte concernée s'est rendue chez sa gynécologue après la douzième semaine de sa grossesse. La gynécologue savait que le fotus de sexe féminin courrait un risque accru d'être atteint d'un trouble du métabolisme héréditaire. Elle aurait donc dû procéder à des examens prénataux plus poussés sur cette femme, ce qu'elle n'a pas fait. L'instance précédente [2] ${ }^{*}$ avait constaté que la mère aurait très vraisemblablement avorté si elle avait été informée de la maladie de sa fille. Elle a admis la demande de réparation morale de la mère, mais rejeté celle de l'enfant au motif que la comparaison entre la vie d'une personne handicapée et la vie que celle-ci aurait hypothétiquement menée sans son handicap n'était pas recevable. Les demanderesses (mère et fille) ont recouru auprès de la Cour suprême du canton de Berne. Les paragraphes suivants exposent les raisons pour lesquelles, sur la base des mêmes faits, la Cour suprême a statué différemment sur les droits de la mère et ceux de l'enfant, de manière a priori surprenante.

\section{Conditions liées à la responsabilité et réparation morale pour la mère}

Les cas dans lesquels une planification familiale défaillante aboutit à une grossesse non planifiée (wrongful pregnancy, wrongful conception), dans lesquels des conseils erronés en matière de génétique ne permettent pas d'éviter une grossesse, ou dans lesquels un diagnostic prénatal erroné n'aboutit pas à une interruption de grossesse (wrongful birth) donnent régulièrement lieu au versement de dommages-intérêts ou d'indemnités pour tort moral si les conditions liées à la responsabilité sont réalisées et si la mère, et dans certains cas également le père, agit en son propre nom. La question du droit d'un enfant handicapé de naissance à réclamer des dommages et intérêts et une indemnité pour tort moral parce que sa mère n'a pas avorté faute de diagnostic prénatal ou en raison d'un diagnostic prénatal erroné est controversée et trouve plus difficilement réponse (wrongful life).

Un médecin répond en principe de la naissance d'un enfant non désiré si les quatre conditions suivantes sont réalisées [3]:

\section{Préjudice ou dommage immatériel}

Dans le cadre de la naissance d'un enfant non désiré, le préjudice ne réside pas dans l'existence de l'enfant, mais dans les coûts engendrés par son éducation et les soins qu'il nécessite. Le préjudice se calcule selon la théorie de la différence. Le préjudice corporel doit entraîner un dommage patrimonial involontaire. On procède donc à une comparaison de l'état du patrimoine avec et sans l'événement dommageable. Le dommage immatériel (moral) de la mère causé par la naissance d'un enfant handicapé - désiré, mais uniquement dans la mesure où il n'était pas handicapé - donne lieu au versement d'une indemnité à titre de réparation morale. En effet, le fait de ne pas interrompre la grossesse a porté atteinte au droit de la mère à l'autodétermination. Par ailleurs, les soins requis par l'enfant constituent une atteinte à la mère, tant en ce qui concerne le mode de vie de cette dernière qu'en ce qui concerne sa relation avec l'enfant.

\section{Violation du contrat}

La femme enceinte a conclu avec sa gynécologue un contrat de soins impliquant un traitement diligent. Un tel traitement inclut également un diagnostic prénatal lorsqu'il est indiqué, pour autant que la femme enceinte n'y renonce pas d'elle-même après avoir reçu des éclaircissements suffisants. Si le médecin ne procède pas au diagnostic indiqué, il ne traite pas sa patiente de manière diligente et viole le contrat.

\section{Rapport de causalité}

Il existe un rapport de causalité entre l'absence de diagnostic prénatal et la naissance d'un enfant handicapé si un traitement diligent avait pu éviter cette naissance. Lorsque le foetus est atteint d'un handicap, le rapport de causalité ne peut exister que si l'interruption de grossesse était légale et si la femme enceinte, ayant connaissance de ce handicap, avait interrompu sa grossesse [4]. Si ces deux conditions ne sont pas réalisées, la grossesse aurait abouti à la naissance d'un enfant handicapé même si le médecin avait procédé au diagnostic de manière diligente. Dans le cas jugé, la Cour a considéré que l'indication médicale de l'art. 119 al. 1 CP était réalisée, car la 
mère était tombée dans un état de détresse profonde en raison de sa grossesse et de la naissance de l'enfant handicapé. Manifestement, la mère a pu établir qu'elle aurait avorté si elle avait eu connaissance du handicap. Une telle preuve est en principe admise si le comportement antérieur de la femme concernée ou ses convictions religieuses ne permettent pas d'aboutir à une conclusion contraire (par ex. si elle n'a pas avorté dans une situation comparable par le passé).

\section{Faute}

Dans le cadre des rapports contractuels, l'existence d'une faute est présumée. En l'espèce, la gynécologue n'a pas réussi à renverser cette présomption. Les quatre conditions liées à l'admission de la responsabilité étant réalisées, le droit de la mère à une indemnité pour réparation morale a été admis.

\section{Droit de l'enfant}

La discussion relative au droit de l'enfant handicapé se focalise sur la définition du dommage [5] (comparaison entre la vie avec le handicap concerné et la non-existence) et la violation contractuelle ou la lésion d'un bien juridique (intégrité physique et droit à la non-existence).

\section{Doctrine et jurisprudence}

Dans la doctrine, le droit de l'enfant fait l'objet de controverses. Certains juristes [6] considèrent que le devoir du médecin de procéder au diagnostic prénatal s'étend à l'enfant à naître, car les biens juridiques futurs de ce dernier sont directement concernés. L'interruption de grossesse admise par le droit suisse prévoit clairement que la naissance d'un enfant handicapé peut être perçue comme un préjudice par la mère. L'enfant handicapé peut donc avoir la même perception. Pour des raisons d'équité et d'égalité de traitement, l'enfant se voit octroyer le droit de demander la réparation de son dommage [7]. Il est également mentionné que, si la mère est en droit de se déterminer après avoir évalué les intérêts en jeu (caractère relatif de la vie de l'enfant), l'enfant en revanche n'a aucun droit à ne pas naître, et ce même s'il est probable qu'un handicap rendra sa vie insupportable (caractère absolu de la vie de l'enfant) [8]. Il y a lieu de souligner à cet égard que l'interruption de grossesse est indiquée sur le plan médical si un risque d'atteinte physique grave menace la mère ou si celle-ci se trouve dans un état de détresse profonde. Si l'enfant se voyait octroyer le droit à être avorté, il conviendrait alors de déterminer si son handicap rendrait sa vie insupportable. Une telle appréciation s'avérerait manifestement extrêmement difficile pour un juge ou pour la mère, la question du risque d'atteinte menaçant la mère étant sensiblement plus facile à résoudre en comparaison. Une indemnité pour tort moral ne peut être versée à l'enfant que si le droit à ne pas naître est reconnu à l'enfant handicapé [9]. Or, divers auteurs nient l'existence d'un tel droit [10].

La jurisprudence des Etats voisins varie sur ce point. Seule la France [11] a reconnu à plusieurs reprises un tel droit de l'enfant. Par la suite, la France a édicté une loi prévoyant que personne ne peut réclamer des dommages et intérêts du seul fait de sa naissance [12]. Une réglementation légale analogue existe également en Angleterre [13]. Avant l'entrée en vigueur de cette loi, les tribunaux anglais avaient rejeté une telle demande au motif qu'il n'était pas possible de calculer le dommage en se fondant sur la théorie de la différence [14]. Un jugement similaire a été rendu en Autriche [15] pour les mêmes motifs. Le Bundesgerichtshof allemand a lui aussi nié l'existence d'un tel droit à l'enfant handicapé [16]. Selon lui, l'enfant ne saurait alléguer qu'il aurait mieux valu qu'il ne vienne pas au monde.

\section{Motifs de l'arrêt de la Cour suprême du canton de Berne}

La Cour suprême [17] du canton de Berne a retenu que l'une des conditions pour qu'il y ait dommage immatériel, et par conséquent pour qu'une indemnité pour tort moral soit versée, est la violation du droit à l'intégrité physique. En l'espèce, la gynécologue n'a pas porté atteinte à l'intégrité physique de l'enfant et il n'existait aucune possibilité d'influer sur la maladie due à des facteurs génétiques. Il n'y a donc pas de lésion corporelle. On ne saurait faire abstraction du fait que la seule alternative aurait été la non-existence de l'enfant. La notion d'atteinte implique une comparaison avec un état alternatif plus favorable. Compte tenu du fait qu'une vie dénuée d'handicap n'aurait de toute manière pas été possible, il n'existe pas d'autre alternative que la nonexistence. Considérant qu'il n'était pas possible de dissocier le handicap de la vie de l'enfant, la Cour a nié l'existence d'un dommage corporel causé par la gynécologue et, par conséquent, d'un dommage matériel.

La Cour a ensuite examiné s'il était possible de déduire un droit à la réparation morale d'une violation d'un droit subjectif à ne pas naître. Elle est parvenue à la conclusion que si la Suisse octroyait aux mères le droit d'avorter dans certains cas, le droit suisse ne connaissait pas de droit de l'enfant à ne pas accéder à la vie. Contrairement à sa mère, l'enfant à naître n'a pas le droit de choisir si la grossesse doit être interrompue ou non, le droit de la mère ne permettant pas de déduire un droit de l'enfant à ne pas naître. La gynécologue n'avait pas davantage d'obligation contractuelle envers l'enfant de ne pas le laisser venir au monde.

La Cour suprême a rejeté la demande de l'enfant en réparation morale. Aucun recours n'a été déposé au Tribunal fédéral contre cet arrêt qui est définitif et exécutoire. Le Tribunal fédéral n'a pas encore eu à se prononcer sur de tels cas. 


\section{Références}

1 Arrêt du 2 mai 2011 de la 2e Chambre civile de la Cour suprême du canton de Berne.

2 Jugement du 18 octobre 2010 du président du Tribunal d'arrondissement judiciaire VIII Berne-Laupen.

3 Art. 41 CO en relation avec l'art. 97 et l'art. 298 CO.

4 Pally U. Arzthaftung mit den Schwerpunkten Schwangerschaftsbetreuung und Geburtshilfe, thèse Zurich 2007, 53 s. et les références citées.

5 Pally, 25 ss; Henri Torrione, Le Préjudice d'être né, REAS 2006, 388 ss.

6 Mannsdorfer TM. Pränatale Schädigung, thèse Fribourg 2000, n. 931 ss; Christoph Müller, Arzthaftung für das unerwünschte behinderte Kind, PJA 5/2003, 522 ss, 530 ss

7 Chappuis G. L'indemnisation du préjudice découlant de la naissance d'un enfant non désiré: une simple question d'arithmétique? Quelques considérations à la lumière des solutions apportées par le droit français, REAS 2006, 378 ss, 381 et n. 22.

8 Picker E. Schadenersatz für das unerwünschte eigene Leben, «Wrongful life», Tubingue 1995, 40 ss.

9 Claudia Junker, Pflichtverletzung, Kindesexistenz und Schadenersatz, Berlin 2002, thèse Heidelberg 2000, 643,645 s., 654,660 ss.
10 Melchert T. Ärztliche Haftpflicht bei Fällen von Wrongful life, Jusletter 12.1.2004, n. 2.

11 Arrêt de la Cour de cassation du 17 novembre 2000, JCP G 2000, II, 10438, 2293-2302.

12 Mannsdorfer T. Franz. Kassationshof, 28.11.2001, Zulässigkeit von Schadenersatz- und Genugtuungsansprüchen gegen sorgfaltswidrig handelnde Medizinalpersonen aufgrund der eigenen, von den Eltern aber «unerwünschen» Geburt, PJA 11/2002, 1360 à 1366, 1365 .

13 Rüetschi D. «Wrongful Life» - die französische Sichtweise, FamPra.ch 2/2001, p. 266 ss, 271.

14 McKay v. Essex AHA, (1982) QB 1166, 1192.

15 OHG 1OB91/99k du 25 mai 1999.

16 BGH, NJW 2002, 2636; BGH, VersR 1981, 481, die Alternative, das Leben des Kindes nicht entstehen zu lassen, haben nur die Eltern, nicht aber das Kind. Diese Beurteilung hat der BGH im Zusammenhang mit der Geltendmachung von Schadenersatz eines gesunden ungewollten Kindes vorgenommen.

17 Arrêt de la $2^{\mathrm{e}}$ Chambre civile de la Cour suprême du canton de Berne du 2 mai 2011, cons. C. ii. 